\title{
Local Atomic Structure Analysis of Zr-Ni and Zr-Cu Metallic Glasses Using Electron Diffraction
}

\author{
Akihiko Hirata $^{1, *}$, Takuro Morino ${ }^{1}$, Yoshihiko Hirotsu ${ }^{1}$, Keiji Itoh $^{2}$ and Toshiharu Fukunaga ${ }^{2}$ \\ ${ }^{1}$ Institute of Scientific and Industrial Research, Osaka University, Ibaraki 567-0047, Japan \\ ${ }^{2}$ Research Reactor Institute, Kyoto University, Kumatori-cho, Sennan-gun, Osaka 590-0494, Japan
}

\begin{abstract}
Local atomic structures in $\mathrm{Zr}_{66.7} \mathrm{Ni}_{33.3}$ and $\mathrm{Zr}_{66.7} \mathrm{Cu}_{33.3}$ metallic glasses were examined by using nanobeam electron diffraction (NBED), energy-filtered selected area electron diffraction (SAED) and high-resolution electron microscopy (HREM). Locally ordered regions of atomic medium range order (MRO) were observed in both of the specimens by NBED, although it was difficult to recognize the regions using HREM. Statistical analyses for NBED patterns revealed such a difference in the extended MRO regions between the specimens that the MRO structure in $\mathrm{Zr}_{66.7} \mathrm{Ni}_{33.3}$ is more complex with a large dispersion of interplanar spacings than those in $\mathrm{Zr}_{66.7} \mathrm{Cu}_{33.3}$. To understand nearest-neighbor atomic coordination, we performed electron intensity analyses using energy-filtered SAED patterns and constructed structure models including about

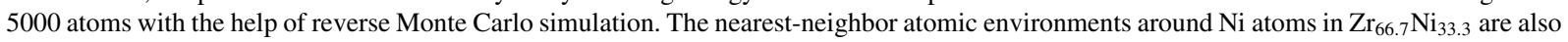
different from those around $\mathrm{Cu}$ atoms in $\mathrm{Zr}_{66.7} \mathrm{Cu}_{33.3}$, consistent with the NBED study. The local structural difference between the two glasses was discussed in relation to their glass-forming abilities. [doi:10.2320/matertrans.MF200618]
\end{abstract}

(Received December 12, 2006; Accepted April 13, 2007; Published May 25, 2007)

Keywords: metallic glass, nanobeam electron diffraction, energy-filtered selected area electron diffraction, reverse Monte Carlo simulation, short range order, medium range order

\section{Introduction}

In recent years, bulk metallic glasses (BMG) have been paid much attention for their promising applications with various characteristic properties. Many attempts have been made to clarify the origin of the extremely high glass stability of the BMGs. To understand the origin, it is significant to study local atomic structures in the as-quenched state and also annealed states. ${ }^{1)}$ It should be noted that the local atomic order can be classified into two-types of atomic order which are called short range order (SRO) and medium range order (MRO). According to the Elliott's definition, ${ }^{2)}$ moreover, the MRO structure has some hierarchies which are short-range MRO $(\sim 0.5 \mathrm{~nm})$, intermediate-range MRO $(0.5 \sim 1 \mathrm{~nm})$, and long-range MRO (1 $\mathrm{nm} \sim)$. SRO and short-range MRO structures, which correspond to the first- and second-nearest neighbor atomic coordinations, have been revealed mainly by using neutron and X-ray diffraction methods through a pair distribution function (PDF) analysis ${ }^{3)}$ or reverse Monte Carlo (RMC) simulation. ${ }^{4}$

In several metal-metalloid systems, Hirotsu and collaborators have been examining local ordered regions extended up to $1 \sim 2 \mathrm{~nm}$ with bcc and fcc structures by means of highresolution electron microscopy (HREM) and nanobeam electron diffraction (NBED) techniques. ${ }^{5-10)}$ The extended ordered regions has been considered to correspond to the long-range MRO (LR-MRO) structure defined by Elliott. ${ }^{2)}$ In metal-metal systems, on the other hand, LR-MRO structures have not been studied well to our best knowledge, although icosahedral-like SRO or MRO structure has been proposed. ${ }^{11-15)}$ When the LR-MRO structure becomes complex, it is difficult to clarify the structural features from the HREM images. In this study, we tried to reveal LR-MRO structures in $\mathrm{Zr}_{66.7} \mathrm{Ni}_{33.3}$ and $\mathrm{Zr}_{66.7} \mathrm{Cu}_{33.3}$ metallic glasses by using NBED and HREM techniques. Note that these two glasses

*Corresponding author, E-mail: ahirata@sankan.osaka-u.ac.jp show different behaviors on annealing with a normal heating rate. The $\mathrm{Zr}_{66.7} \mathrm{Ni}_{33.3}$ glass crystallizes without showing glass transition, whereas the $\mathrm{Zr}_{66.7} \mathrm{Cu}_{33}$ glass shows a clear glass transition before crystallization. Detailed structural studies can provide useful information to understand the reason for the difference in their glass stabilities. In addition to the LRMRO studies by NBED experiments, we also examined local atomic coordinations (SRO structures) for both the glasses by means of selected area electron diffraction (SAED) structure analysis with a help of RMC structural modeling. The glassforming abilities of these glasses were discussed based on the characteristics of observed LR-MRO and SRO structures.

\section{Experimental}

The $\mathrm{Zr}_{66.7} \mathrm{Ni}_{33.3}$ and $\mathrm{Zr}_{66.7} \mathrm{Cu}_{33.3}$ glasses were made by a single-roll rapid quenching technique. The physical densities for $\mathrm{Zr}_{66.7} \mathrm{Ni}_{33.3}$ and $\mathrm{Zr}_{66.7} \mathrm{Cu}_{33.3}$ used in this study are 6.84 $\mathrm{g} / \mathrm{cm}^{3}$ and $7.06 \mathrm{~g} / \mathrm{cm}^{3}$, respectively. ${ }^{4}$ ) The samples were thinned using low-angle ion-milling (Gatan, PIPS) and finally polished with low-voltage ion-milling (TECHNOORG LINDA, IV3/L). NBED patterns and HREM images were taken by using a JEM-3010 TEM operated at $300 \mathrm{kV}$. Energy-filtered SAED patterns for structure analyses were obtained by using energy-filtering TEM (LEO-922D operated at $200 \mathrm{kV}$ ) equipped with an in-column type energy filter. The inelastic part of intensity was eliminated using an energy window of about $20 \mathrm{eV}$. The NBED patterns were recorded using a TV-rate CCD camera. The SAED patterns and HREM images were recorded on imaging plates.

\section{Results}

Figures 1(a) and (b) show HREM images obtained from $\mathrm{Zr}_{66.7} \mathrm{Ni}_{33.3}$ and $\mathrm{Zr}_{66.7} \mathrm{Cu}_{33.3}$, together with the corresponding SAED patterns. The defocus value in taking the images was about $60 \mathrm{~nm}$ (underfocus), which condition provides clear 

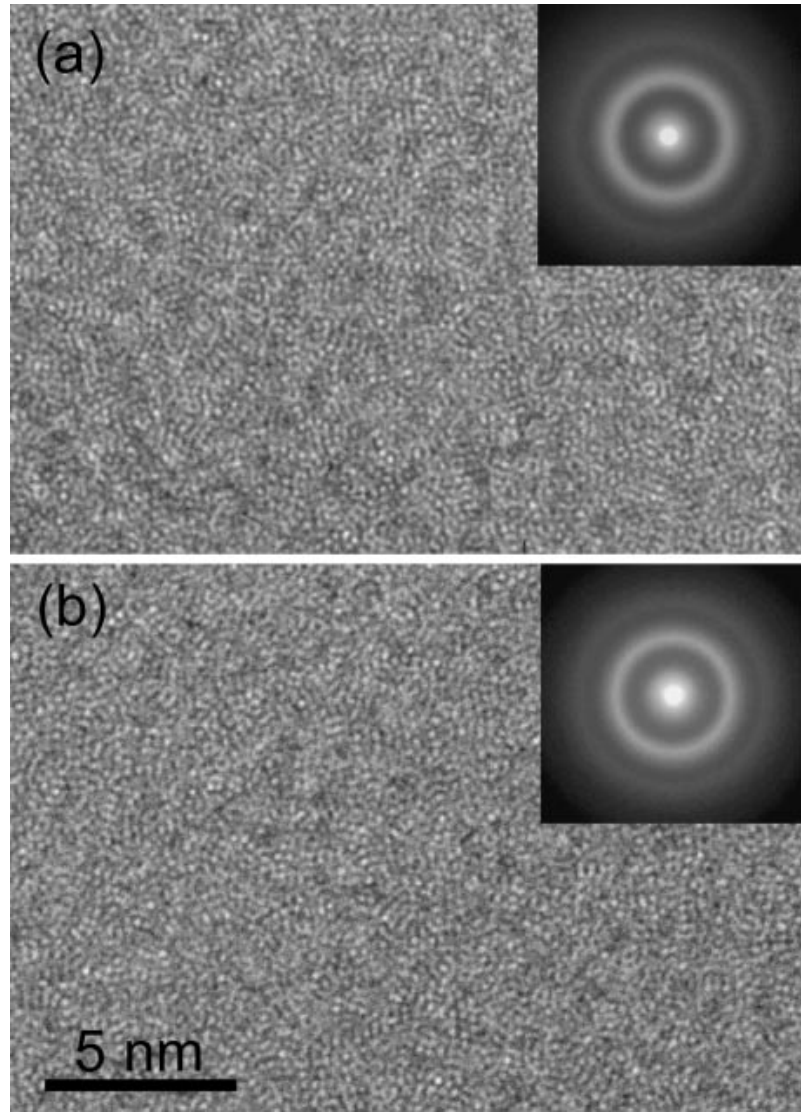

Fig. 1 High-resolution electron micrographs obtained from (a) $\mathrm{Zr}_{66.7} \mathrm{Ni}_{33.3}$ and (b) $\mathrm{Zr}_{66.7} \mathrm{Cu}_{33.3}$, together with corresponding selected area electron diffraction patterns. The defocus value in taking the images is about $-60 \mathrm{~nm}$.

images of local ordered regions. The corresponding SAED patterns show fully halo patterns without crystalline reflections. The "salt and pepper" contrasts typical of amorphous structures were observed in the HREM images of these glasses. However, it is difficult to detect the local ordered regions with crossed straight lattice fringes, which are clearly observed in Pd- and Fe-based metallic glasses. ${ }^{5-10)}$ In the present case, we cannot judge the presence of the local ordered regions from the HREM images.

The presence of the ordered regions can be confirmed by taking the NBED patterns with a fine electron probe (a full width at half maximum of the probe is about $1 \mathrm{~nm}$ ), although they cannot be recognized in the HREM images. Typical NBED patterns taken from $\mathrm{Zr}_{66.7} \mathrm{Ni}_{33.3}$ and $\mathrm{Zr}_{66.7} \mathrm{Cu}_{33.3}$ are shown in Fig. 2(a) and (b), respectively. Although the SAED patterns show fully halo patterns, crystalline spots with relatively strong intensity are found just on a position of the first halo rings in NBED patterns. Since such spots cannot be observed from a dense random packing (DRP) structure model ${ }^{16)}$ the LR-MRO regions are judged to be included in both of the glasses.

In order to obtain structural information from the LRMRO regions, we examined NBED patterns (more than 200) statistically for each of the glass. In these NBED patterns, we could easily eliminate strong diffraction spots from diffuse dots with weak intensities forming halo-diffraction ring (diffraction spots with intensities two times stronger than
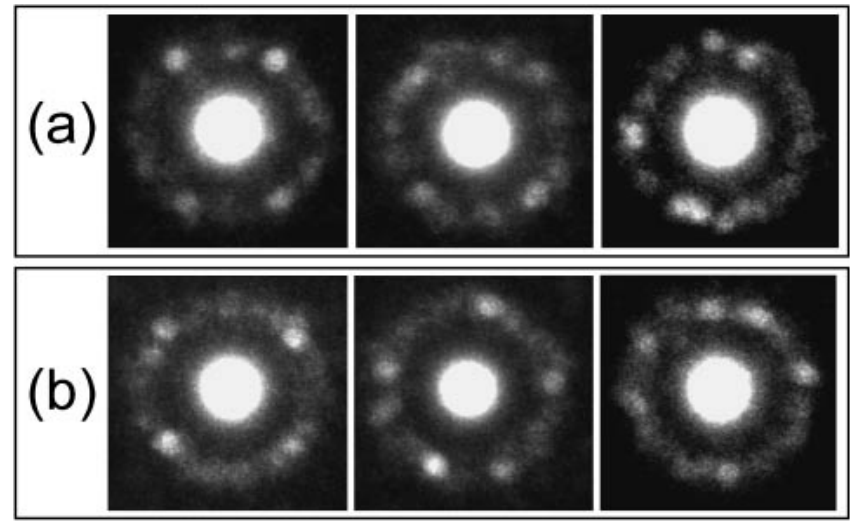

Fig. 2 Nanobeam electron diffraction patterns obtained from (a) $\mathrm{Zr}_{66.7^{-}}$ $\mathrm{Ni}_{33.3}$ and (b) $\mathrm{Zr}_{66.7} \mathrm{Cu}_{33.3}$. A full width at half maximum of electron probe used in taking the patterns is about $1 \mathrm{~nm}$.
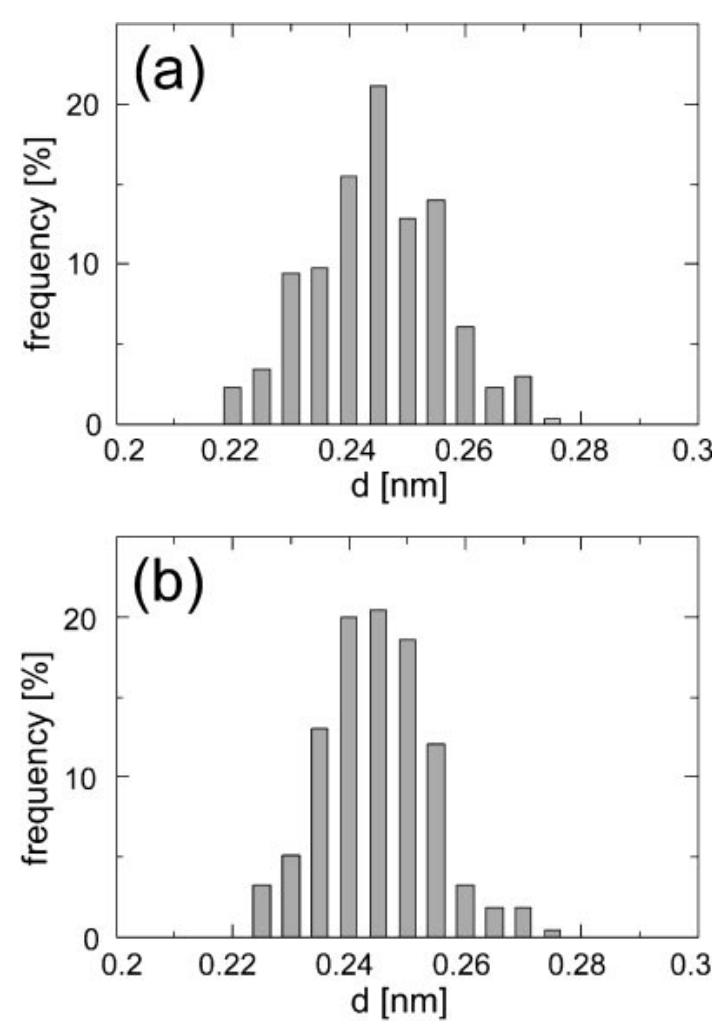

Fig. 3 Frequency distributions of reciprocal lengths between a direct beam and strong spots in nanobeam electron diffraction patterns for (a) $\mathrm{Zr}_{66.7}$ - $^{-}$ $\mathrm{Ni}_{33.3}$ and (b) $\mathrm{Zr}_{66.7} \mathrm{Cu}_{33.3}$.

those of the diffuse dots were chosen). Figure 3 shows the frequency histograms of interplanar spacings (hereafter we call them d-spacings) of the LR-MRO regions obtained from the NBED analyses for $\mathrm{Zr}_{66.7} \mathrm{Ni}_{33.3}$ and $\mathrm{Zr}_{66.7} \mathrm{Cu}_{33.3}$. Note that " 0.24 " of the $\mathrm{d}$-spacing shows a range between 0.235 $0.240 \mathrm{~nm}$ in the histograms. By using a standard specimen (a gold particle), the error of $d$ value was estimated to be about $\pm 0.005 \mathrm{~nm}$. The dispersion of $\mathrm{d}$-spacings for $\mathrm{Zr}_{66.7} \mathrm{Ni}_{33.3}$ is wider than that for $\mathrm{Zr}_{66.7} \mathrm{Cu}_{33.3}$, although both of the distributions are basically centered around $0.240 \sim 0.245 \mathrm{~nm}$.

In order to know SRO structures of the glasses, we also performed average structure analyses using energy-filtered SAED obtained from large selected areas $(400 \mathrm{~nm}$ in 


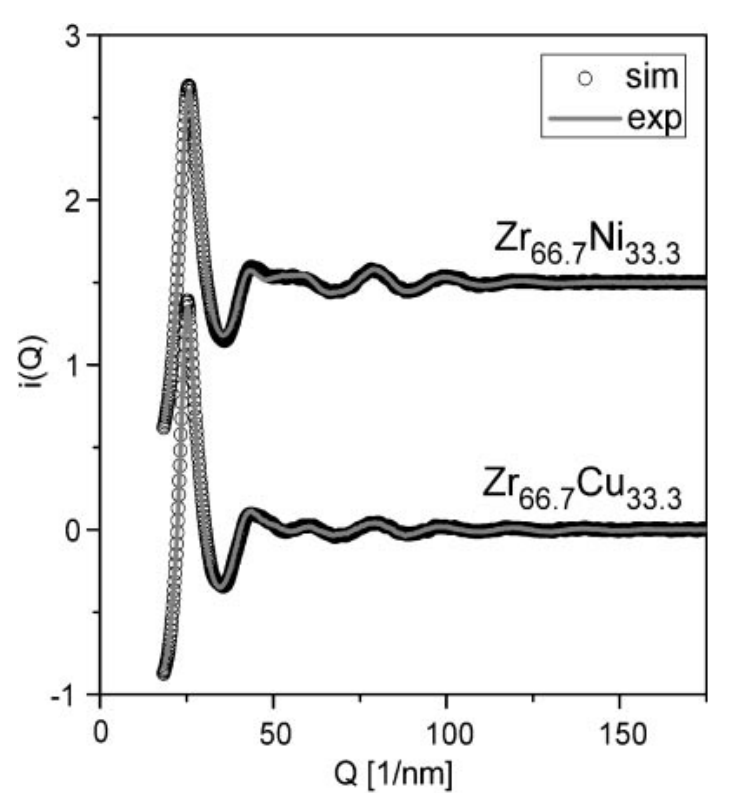

Fig. 4 Experimental interference function $(\mathrm{i}(Q))$ profiles of $\mathrm{Zr}_{66.7} \mathrm{Ni}_{33.3}$ and $\mathrm{Zr}_{66.7} \mathrm{Cu}_{33.3}$ fitted to simulated profiles.

diameter). Plausible structure models were constructed using RMC simulation ${ }^{17)}$ for both of the glasses. In constructing each structure model, we initially made a dense random packing model with 4999 atoms by static relaxation with Lennard-Jones atomic potentials which could reproduce expected inter-atomic distances of constituent atoms. Then, the RMC simulation was performed to fit the experimental interference function $(\mathrm{i}(Q))$ to the experimental one. The weighting factors for each partial i $(Q)$ used in the simulation were taken to be dependent on the $Q$-vector. Figure 4 show the RMC simulated $\mathrm{i}(Q)$ profiles obtained from the final structure models for $\mathrm{Zr}_{66.7} \mathrm{Ni}_{33.3}$ and $\mathrm{Zr}_{66.7} \mathrm{Cu}_{33.3}$. A small difference between these profiles is found: the $\mathrm{i}(Q)$ profile of $\mathrm{Zr}_{66.7} \mathrm{Ni}_{33.3}$ dumps more slowly than that of $\mathrm{Zr}_{66.7} \mathrm{Cu}_{33.3}$. In order to understand local atomic structures, we next calculated partial-PDFs and performed Voronoi polyhedral analyses for the final structure models. Figure 5 shows partial PDFs for these glasses derived from their structure models together with the total PDFs. A split of the first peak in $\mathrm{Zr}_{66.7} \mathrm{Ni}_{33.3}$ is found to be larger than that in $\mathrm{Zr}_{66.7} \mathrm{Cu}_{33.3}$, indicating a difference in local atomic environment. Such characteristic difference in PDF profiles between the $\mathrm{Zr}-\mathrm{Cu}$ and $\mathrm{Zr}$-Ni glasses were also pointed out by Saida et al. ${ }^{18)}$

Figure 6 shows histograms for numbers of Voronoi polyhedra surrounding $\mathrm{Zr}$ atoms in $\mathrm{Zr}_{66.7} \mathrm{Ni}_{33.3}$ and $\mathrm{Zr}_{66.7^{-}}$ $\mathrm{Cu}_{33.3}$. Note that total atomic numbers of $\mathrm{Zr}$ and $\mathrm{Ni}(\mathrm{Cu})$ atoms are 3333 and 1666, respectively. A notation of the Voronoi index was already explained in the literature. ${ }^{19)}$ Around $\mathrm{Zr}$ atoms, types and fractions of the polyhedra are very similar between both of the glasses, where indices of " 0 36400 " and "0 110200 " for high-coordination polyhedra are frequently found. Types of polyhedra around $\mathrm{Ni}$ atoms in $\mathrm{Zr}_{66.7} \mathrm{Ni}_{33.3}$, on the other hand, are quite different from those around $\mathrm{Cu}$ atoms in $\mathrm{Zr}_{66.7} \mathrm{Cu}_{33.3}$. The histograms around $\mathrm{Ni}$ and $\mathrm{Cu}$ atoms are shown in Fig. 7(a) and (b). Around $\mathrm{Ni}$ atoms, a trigonal prism polyhedron denoted by "0 36000 " dominates and average coordination number is less than 10 .
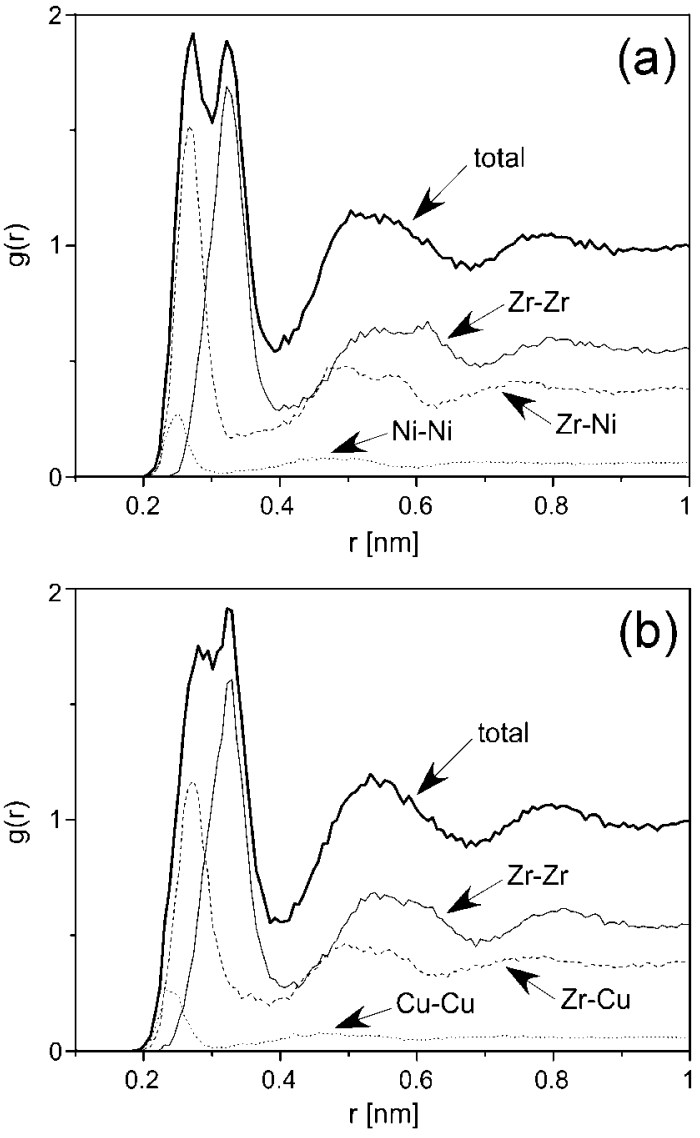

Fig. 5 Total and partial pair distribution functions obtained from final structure models of (a) $\mathrm{Zr}_{66.7} \mathrm{Ni}_{33.3}$ and (b) $\mathrm{Zr}_{66.7} \mathrm{Cu}_{33.3}$.
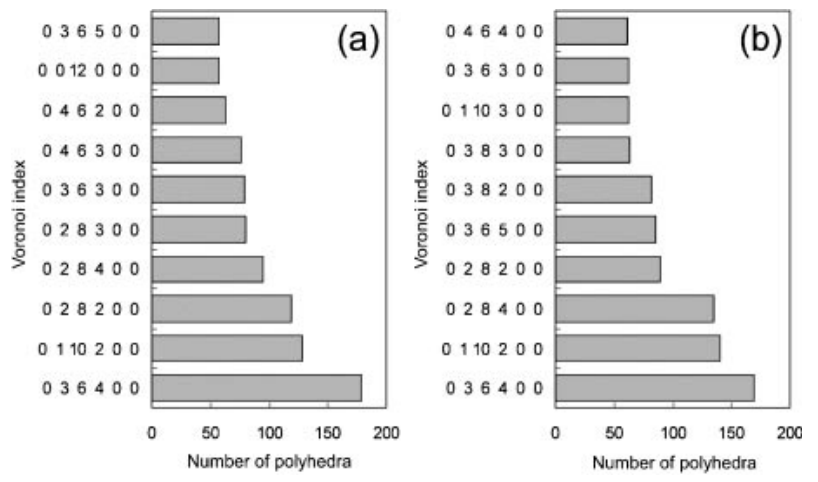

Fig. 6 Voronoi polyhedra around $\mathrm{Zr}$ atoms for (a) $\mathrm{Zr}_{66.7} \mathrm{Ni}_{33.3}$ and (b) $\mathrm{Zr}_{66.7} \mathrm{Cu}_{33.3}$.

Most of polyhedra with central $\mathrm{Cu}$ atoms, on the other hand, are relatively close to icosahedral-like coordination of "0 28 100 " and the average coordination number approximates to 11 .

\section{Discussion}

In this study, we performed local structure analyses for the $\mathrm{Zr}_{66.7} \mathrm{Ni}_{33.3}$ and $\mathrm{Zr}_{66.7} \mathrm{Cu}_{33.3}$ metallic glasses. The NBED study revealed the presence of the LR-MRO structures for both the alloys and the characteristic difference in the dspacing distributions of the LR-MRO structures. The average structure analyses were also performed on the basis of SAED 

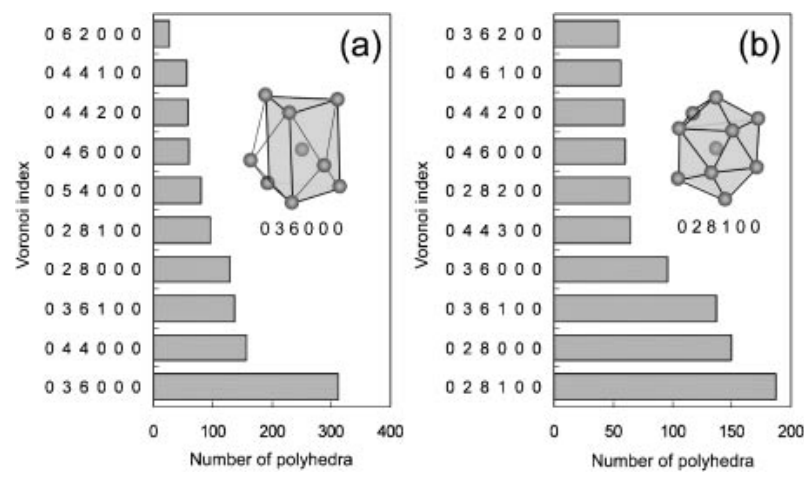

Fig. 7 Voronoi polyhedra around (a) $\mathrm{Ni}$ atoms for $\mathrm{Zr}_{66.7} \mathrm{Ni}_{33.3}$ and (b) $\mathrm{Cu}$ atoms for $\mathrm{Zr}_{66.7} \mathrm{Cu}_{33.3}$.

intensity analyses to reveal the SRO structures. In the following, we discuss a structural relation between the SRO and LR-MRO structures in the present metallic glasses, together with related crystalline structures.

The RMC-simulated structure models reveal that the local atomic coordinations around smaller atoms $(\mathrm{Cu}$ and $\mathrm{Ni})$ are different, although those around large $\mathrm{Zr}$ atoms are very similar. Around smaller atoms, $\mathrm{Zr}_{66.7} \mathrm{Cu}_{33.3}$ forms more isotropic SRO structures in comparison with the case of $\mathrm{Zr}_{66.7} \mathrm{Ni}_{33.3}$, because many icosahedral-like coordination polyhedra appear in $\mathrm{Zr}_{66.7} \mathrm{Cu}_{33.3}$. In $\mathrm{Zr}_{66.7} \mathrm{Ni}_{33.3}$, on the other hand, the predominant SRO structure is prism-type, which is relatively anisotropic structure compared with the icosahedral-like one. The above characteristics with the difference of coordination polyhedra are well consistent with a previous result obtained from a RMC simulation fitted to X-ray and neutron diffraction profiles. ${ }^{4)}$ The statistical analyses of NBED patterns for these glasses show a difference in their dispersions of d-spacings, indicating that the LR-MRO structures are different between $\mathrm{Zr}_{66.7} \mathrm{Ni}_{33.3}$ and $\mathrm{Zr}_{66.7} \mathrm{Cu}_{33.3}$, although both of the dispersions are centered around $0.240 \sim 0.245 \mathrm{~nm}$. A broad d-spacing dispersion of LR-MROs in $\mathrm{Zr}_{66.7} \mathrm{Ni}_{33.3}$ is indicative of a presence of LR-MRO regions with relatively anisotropic or complex structures as well as the SRO structure mentioned above. The main d-spacings with strong diffraction intensities found in the tetragonal $\mathrm{Zr}_{2} \mathrm{Ni}$ phase, which is known as the primary phase in crystallization $^{20)}$ and consists of prism-type structural units, are distributed widely as $0.254 \mathrm{~nm}$ (for $\{211\}$ planes), $0.229 \mathrm{~nm}(\{220\})$, and $0.228 \mathrm{~nm}(\{112\})$. The wide d-spacing dispersion of LR-MROs in $\mathrm{Zr}_{66.7} \mathrm{Ni}_{33.3}$ is probably ascribed to LR-MRO structures which are partially similar to the $\mathrm{Zr}_{2} \mathrm{Ni}$ structure. It should be noted, however, that there are no planes in the crystal structure of $\mathrm{Zr}_{2} \mathrm{Ni}$, which correspond to most frequent d-spacing found in Fig. 3(a) $(0.240 \sim 0.245$ $\mathrm{nm})$. The LR-MRO structures in $\mathrm{Zr}_{66.7} \mathrm{Ni}_{33.3}$ are presumed to be deformed from the $\mathrm{Zr}_{2} \mathrm{Ni}$ structure or partially consist of isotropic $\mathrm{Zr}$-Ni clusters with d-spacings near $0.240 \sim 0.245$ $\mathrm{nm}$. As for $\mathrm{Zr}_{66.7} \mathrm{Cu}_{33.3}$, on the other hand, the dispersion is relatively small, indicating that the LR-MRO structures are rather isotropic or simple as well as the SRO structure. The tetragonal $\mathrm{Zr}_{2} \mathrm{Cu}$ crystalline phase which appears in the primary crystallization of the $\mathrm{Zr}_{66.7} \mathrm{Cu}_{33.3}$ glass ${ }^{21)}$ has a tetragonal symmetry and main d-spacings in $\mathrm{Zr}_{2} \mathrm{Cu}$ are
$0.244 \mathrm{~nm}$ (for $\{103\}$ planes) and $0.228 \mathrm{~nm}(\{110\})$. Although the distribution of those main d-spacings for $\{103\}$ in $\mathrm{Zr}_{2} \mathrm{Cu}$ corresponds to that of the d-spacings observed by NBED for $\mathrm{Zr}_{66.7} \mathrm{Cu}_{33.3}$, the frequency for $\mathrm{d}$-spacing for $\{110\}$ is found to be extremely small, indicating that the LR-MRO structure of $\mathrm{Zr}_{66.7} \mathrm{Cu}_{33.3}$ does not seem to include the structural component of the tetragonal $\mathrm{Zr}_{2} \mathrm{Cu}$ phase.

In the crystallization process of glass, the LR-MRO structure is considered to play an important role as a preliminary structure for nucleus. When the LR-MRO structure is similar to the structure for the primary crystallization, the crystallization is probably easily promoted kinetically. In the present study, the LR-MRO structure in $\mathrm{Zr}_{66.7} \mathrm{Ni}_{33.3}$ is considered to be relatively similar to the $\mathrm{Zr}_{2} \mathrm{Ni}$ structure, whereas there is no strong correlation between the LR-MRO structure in $\mathrm{Zr}_{66.7} \mathrm{Cu}_{33.3}$ and the $\mathrm{Zr}_{2} \mathrm{Cu}$ structure. During annealing, as was mentioned earlier, $\mathrm{Zr}_{66.7} \mathrm{Cu}_{33.3}$ shows a clear glass transition before crystallization, while $\mathrm{Zr}_{66.7} \mathrm{Ni}_{33.3}$ directly crystallizes into $\mathrm{Zr}_{2} \mathrm{Ni}$. The structural similarity between the LR-MRO and the primary phase in crystallization is judged to be one of the important factors to determine glass stability.

\section{Conclusions}

We examined LR-MRO structures in Zr-based metallic glasses by using NBED and HREM techniques, together with SAED intensity analysis with a help of RMC simulation. To clarify characteristics of the LR-MRO regions, we analyzed statistical frequencies of positions of the strong diffraction spots in NBED patterns obtained from MRO regions. It was found that the dispersion of the NBED spot positions (corresponding to the $\mathrm{d}$-spacing) in $\mathrm{Zr}_{66.7} \mathrm{Ni}_{33.3}$ is broader than that in $\mathrm{Zr}_{66.7} \mathrm{Cu}_{33.3}$. From this result, it was expected that a MRO structure with a low structural symmetry similar to that of a tetragonal $\mathrm{Zr}_{2} \mathrm{Ni}$ (the primary phase in the crystallization) is formed in $\mathrm{Zr}_{66.7} \mathrm{Ni}_{33.3}$ and influences to the crystallization process. Whereas, from the dispersion of NBED spot positions, $\mathrm{Zr}_{66.7} \mathrm{Cu}_{33.3}$ was judged to have more isotropic MRO structures in comparison with $\mathrm{Zr}_{66.7} \mathrm{Ni}_{33.3}$. These local structures expected from the NBED results are well consistent with those of local coordination polyhedra extracted from the simulated structural models based on halodiffraction intensity profile analyses.

\section{Acknowledgement}

This work is partly supported by the Grant in Aid of Ministry of Education, Sports, Culture, Science and Technology, Priority Area on "Materials Science of Bulk Metallic Glasses" and by the Ministry of Education, Science, Sports and Culture, Grant-in-Aid for Scientific Research (B), 18360306, 2006. We acknowledge Dr. M. Hasegawa of Tohoku University for providing samples.

\section{REFERENCES}

1) A. Inoue: Acta Mater. 48 (2000) 279.

2) S. R. Elliott: Physics of amorphous materials, (New York, John Wiley \& Sons, 1990), p. 71. 
3) S. Sato, T. Sanada, J. Saida, M. Imafuku, E. Matsubara and A. Inoue: Mater. Trans. 46 (2005) 2893.

4) T. Fukunaga, K. Itoh, T. Otomo, K. Mori, M. Sugiyama, H. Kato, M. Hasegawa, A. Hirata, Y. Hirotsu, A. C. Hannon: Intermetallics 14 (2006) 893.

5) Y. Hirotsu and R. Akada: Jpn. J. Appl. Phys. 23 (1984) L478.

6) Y. Hirotsu, M. Uehara and M. Ueno: J. Appl. Phys. 59 (1986) 3081.

7) K. Anazawa, Y. Hirotsu and Y. Inoue: Acta Met. Mater. 42 (1994) 1997.

8) Y. Hirotsu, T. Ohkubo and M. Matsushita: Microsc. Res. Tech. 40 (1998) 284.

9) T. Ohkubo and Y. Hirotsu: Phys. Rev. B 67 (2003) 094201.

10) A. Hirata, Y. Hirotsu, E. Matsubara, T. Ohkubo and K. Hono: Phys. Rev. B 74 (2006) 184204.

11) D. Kofalt, S. Nanao, T. Egami, K. M. Wong, and S. J. Poon: Phys. Rev. Lett. 57 (1986) 114.

12) T. Takagi, T. Ohkubo, Y. Hirotsu, B. S. Murty, K. Hono and D. Shindo:
Appl. Phys. Lett. 79 (2001) 485.

13) K. Luo, H. W. Sheng, F. M. Alamgir, J. M. Bai, J. H. He and E. Ma: Phys. Rev. Lett. 92 (2004) 145502.

14) L. Yang, J. Z. Jiang, T. Liu, T. D. Hu and T. Uruga: Appl. Phys. Lett. 87 (2005) 061918.

15) J. Saida, T. Sanada, S. Sato, M. Imafuku, E. Matsubara and A. Inoue: J. Alloy Compd. 434-435 (2007) 135.

16) A. Hirata, Y. Hirotsu, T. Ohkubo, T. Hanada and V. Bengus: Phys. Rev. B 74 (2006) 214206.

17) R. McGreevy, L. Pusztal: Mol. Simulation 1 (1988) 359.

18) J. Saida, E. Matsubara and A. Inoue: Mater. Trans. 44 (2003) 1971.

19) M. Tanemura, Y. Hiwatari, H. Matsuda, T. Ogawa, N. Ogita and A. Ueda: Prog. Theor. Phys. 58 (1977) 1079.

20) T. Nagase, M. Nakamura and Y. Umakoshi: Intermetallics 15 (2007) 211.

21) T. Nagase and Y. Umakoshi: Mater. Sci. Eng. A 343 (2003) 13. 